This article was published in Journal of Global Antimicrobial Resistance, 2, 309-315, 2014

http://dx.doi.org/10.1016/j.jgar.2014.10.001

\title{
blaTEM and vanA as indicator genes of antibiotic resistance contamination in a hospital-urban wastewater treatment plant system
}

Carlos Narciso-da-Rocha a, Ana R. Varela a,b, Thomas Schwartz c, Olga C. Nunes ${ }^{b}$, Célia M. Manaia ${ }^{a, *}$

a CBQF - Centro de Biotecnologia e Química Fina - Laboratório Associado, Escola Superior de Biotecnologia, Universidade Católica Portuguesa/Porto, Rua Arquiteto Lobão Vital, Apartado 2511, Porto 4202-401, Portugal

b Laboratório de Engenharia de Processos Ambiente, Biotecnologia e Energia (LEPABE), Departamento de Engenharia Química, Faculdade de Engenharia, Universidade do Porto, R. Dr Roberto Frias, 4200-465 Porto, Portugal

c Karlsruhe Institute of Technology (KIT), Campus North, Institute of Functional Interfaces (IFG), Microbiology of Natural and Technical Interfaces Department, Herman-von-Helmholtz-Platz 1, 76344 Eggenstein-Leopoldshafen, Germany

\section{Abstract}

Four indicator genes were monitored by quantitative PCR in hospital effluent $(\mathrm{HE})$ and in the raw and treated wastewater of the municipal wastewater treatment plant receiving the hospital discharge. The indicator genes were the class 1 integrase gene intI1, to assess the capacity of bacteria to be involved in horizontal gene transfer processes; blaTEM, one of the most widespread antibiotic resistance genes in the environment, associated with Enterobacteriaceae; vanA, an antibiotic resistance gene uncommon in the environment and frequent in clinical isolates; and marA, part of a locus related to the stress response in Enterobacteriaceae. Variation in the abundance of these genes was analysed as a function of the type of water, and possible correlations with cultivable bacteria, antimicrobial residue concentrations, and bacterial community composition and structure were analysed. $\mathrm{HE}$ was confirmed as an important source of blaTEM and vanA genes, and wastewater treatment showed a limited capacity to remove these resistance genes. The genes blaTEM and vanA presented the strongest correlations with culturable bacteria, antimicrobial residues and some bacterial populations, representing interesting candidates as indicator genes to monitor resistance in environmental samples. The intI1 gene was the most abundant in all samples, demonstrating that wastewater bacterial populations hold a high potential for gene acquisition. 
(C) 2014 International Society for Chemotherapy of Infection and Cancer. Published by Elsevier Ltd. All

\section{Introduction}

Contamination of different water environments with antibiotic residues, antibiotic-resistant bacteria and antibiotic resistance genes is an environmental problem reported in wastewater, coastal water, lakes, rivers, springs, underground water bodies and even tap water [1]. Extensive use of antibiotics and the inevitable discharge of at least part of these residues and resistant bacteria in the municipal sewer are important drivers of this emerging form of contamination [2]. Although urban wastewater treatment plants (UWTPs) are designed to clean water by removing nutrients and pathogenic micro-organisms, they cannot completely eliminate antibiotic residues or antibiotic-resistant bacteria [3]. Unlike antibiotic residues, antibiotic-resistant bacteria proliferate in the environment, spreading their antibiotic resistance genes. There- fore, this form of biological pollution is not restricted to a point source of contamination or occasional discharge [2], persisting and spreading in the environment even in the absence of antibiotic residues [4].

At the moment, our understanding of the factors that drive the propagation of genetic elements related to resistance during wastewater treatment or after its release in the environment is limited. The available literature shows that different antibiotic- resistant populations or genes present distinct distributions or removal rates during wastewater treatment [1]. This work was designed based on the hypothesis that genes related to resistance may present different patterns of variation, which will influence their penetrance as environmental contaminants. To test this hypothesis, the abundance of four genes was monitored over time by quantitative PCR (qPCR) in hospital effluent and in the raw and treated wastewater of the receiving municipal sewage treatment plant. The genes were (i) the class 1 integrase gene intI1, an indicator of the capacity of bacteria to be involved in horizontal gene transfer processes, since it is frequently associated with antibiotic resistance acquisition [5]; (ii) blaTEM, associated mainly with Enterobacteriaceae and common in the environment, as an indicator of anthropogenic antibiotic resistance contamination [6];

(iii) vanA, associated mainly with Enterococcus and uncommon in the environment, as an indicator of antibiotic resistance contamination of clinical origin [7]; and (iv) $m a r A$, associated mainly with Enterobacteriaceae, as an indicator of bacterial stress response capacity [8]. Except for marA, which is part of a polygenic locus on the chromosome, all of these alleles may be inherited vertically or transferred by horizontal gene transfer. Data on the abundance of each indicator gene was analysed as a function of the levels of antibiotic-resistant cultivable bacteria, the 16S rRNA gene-based denaturing gradient gel electrophoresis (DGGE) patterns and the concentrations of antimicrobial residues. It was intended to assess whether: (i) genes related to different functions present distinct abundance and patterns of variation in different types of wastewater; (ii) hospital effluent is a source of resistance genes; and (iii) variations in the abundance of indicator 
genes are significantly correlated with bacterial populations, antibiotic resistance prevalence or antimicrobial residues.

\section{Materials and methods}

\subsection{Sampling and sample characterisation}

This study examined the hospital effluent (HE), raw wastewater (RWW) (inflow) and treated wastewater (TWW) (effluent) of the receiving UWTP described in previous studies [9]. Briefly, the hospital has an average effluent flow of 1000 $\mathrm{m}^{3}$ /day. The receiving ${ }^{3}$ UWTP serves a population equivalent to 200,000 inhabitants and has an average monthly flow of $1.1 \times 10 \mathrm{~m} /$ day. Wastewater treatment comprises preliminary, primary and biological treatment, including nitrogen and phosphorus removal. The treated effluent is discharged to a river mouth.

Sampling and sample characterisation were as described previously [9]. Detailed information is provided in Supplementary Fig. S1 and Supplementary Tables S1 and S2 (for DGGE profiles, CFU counts, and concentrations of antibiotic residues and heavy metals, respectively [9]. Briefly, four grab samples were collected from the HE (one per month in October 2010 and January, February and June 2011), and three 24-h composite samples were obtained from RWW (after the primary settling tank) and TWW (final effluent) of the UWTP in October 2010 and January and February 2011. Samples were characterised for their content of culturable bacteria (total and amoxicillin- and ciprofloxacin-resistant), bacterial community composition based on the 16S rRNA gene-DGGE patterns, and concentration of antibiotic residues and metals, as summarised in Supplementary Tables S1 and S2 and Supplementary Fig. S1 [9].

Supplementary Fig. S1 and Tables S1 and S2 related to this article can be found, in the online version, at doi:10.1016/j.jgar. 2014.10.001.

Total and antibiotic-resistant bacteria were enumerated using the membrane filtration method on plate count agar (PCA) (Pronadisa, Madrid, Spain) for total heterotrophs, on $\mathrm{m}$-faecal coliform agar (mFC) (Difco, Sparks, MD) for enterobacteria, and on glutamate-starch-phenol red agar (GSP) (Merck, Darmstadt, Germany) for aeromonads/pseudomonads. In parallel, bacteria were enumerated on the same media supplemented with $32 \mathrm{mg} / \mathrm{L}$ amoxicillin (Sigma, Steinheim, Germany) or $4 \mathrm{mg} / \mathrm{L}$ ciprofloxacin (Sigma) [3], being considered resistant to the respective antibiotic. All procedures were performed in triplicate (Supplementary Table S1).

Bacterial community characterisation based on DGGE analysis was performed as described previously [3]. Total DNA extracts were obtained in triplicate by filtering $25 \mathrm{~mL}$ of $\mathrm{HE}(n=4)$ or RWW $(n=3)$ and $150 \mathrm{~mL}$ of TWW $(n=3)$ through polycarbonate membranes $(0.2 \mathrm{~mm}$ porosity) (Whatman, Brentford, UK) and total DNA was extracted with a PowerWater $\mathbb{R}$ DNA Isolation Kit (MOBIO Laboratories Inc., Carlsbad, CA). A 180-bp fragment of the 16S rRNA gene was amplified using the primers 338F-GC-clamp and 518R. DGGE was 
performed on a polyacrylamide gel and DGGE profiles were normalised and analysed as described previously (Supplementary Fig. S1) [9]. Selected bands, corresponding to populations whose variation was found to present significant positive correlations with the variation of the studied indicator genes, were identified based on nucleotide sequence analysis, after cloning and GenBank query (http://blast.ncbi.nlm.nih.gov). Samples were also characterised for the content of arsenic, tetracycline, penicillin G, sulfamethoxazole, ciprofloxacin and ofloxacin as described previously [3,9] (Supplementary Table S2).

\subsection{Quantification of indicator genes by real-time PCR}

Real-time PCR was used to assess the abundance (qPCR) of selected genes in the same DNA extracts that were used for DGGE analysis. Three replicates of DNA extract were analysed independently for each sample. Fragments of the genes blaTEM, marA, vanA, intI1 and 16S rRNA were analysed by real-time PCR (StepOne ${ }^{\text {TM }}$ Real-Time PCR System; Life Technologies, Carlsbad, CA) using the

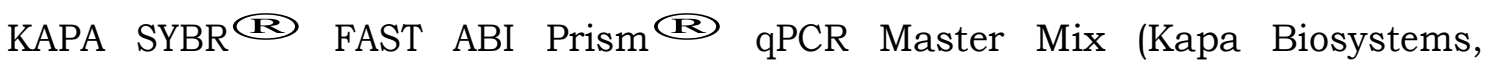
Wilmington, MA) for all targets except vanA, for which the SYBR $\mathbb{R}$ Select Master Mix (Applied Biosystems, Foster City, CA) was used. The primer sets and thermal cycling conditions were as described in Table 1 . The melting curve of the amplicon at increments of $0.1^{\circ} \mathrm{C}$ from $65^{\circ} \mathrm{C}$ to $95^{\circ} \mathrm{C}$ was analysed to assess the homogeneity of the PCR product. Confirmation of the PCR product size was done in a $1.5 \%$ agarose gel. Real-time PCR results were analysed using the StepOne TM v.2.3 software (Life Technologies).

Standard curves for the 16S rRNA and marA amplicons were prepared using the genomic DNA of Escherichia coli ATCC 25992; for blaTEM and intI1, amplicons were prepared as described previously [5,13] using the genomic DNA of E. coli strains M2AC7 (accession no. HG797639) and S3R22 (accession no. HG797640), respectively; amplicons of vanA were obtained from the genomic DNA of Enterococcus faecalis ATCC 17050, as described by Dutka-Malen et al. [14], and were cloned with an InsTAclone TM PCR Cloning Kit (MBI Fermentas). Plasmid DNA was extracted using a GeneJET Plasmid Miniprep Kit (Thermo Fisher Scientific, Waltham, MA) and was treated with Plasmid-Safe TM ATP- Dependent DNase (Epicentre, Madison, WI). All genomic DNA extractions were performed using a QIAamp DNA Stool Kit (QIAGEN, Venlo, The Netherlands) and amplicons were purified using a GRS PCR \& Gel Band Purification Kit (GRISP, Porto, Portugal).

DNA quantification was performed by fluorimetry (Qubit $\mathbb{R}$ Fluorometer; Invitrogen, Carlsbad, CA) as described previously [15]. Ten-fold serial dilutions of purified plasmid DNA were used to prepare standard curves for each indicator gene. Gene copy numbers were calculated by the Standard Curve method as described previously [16]. 


\subsection{Data analyses}

The number of copies of each indicator gene was normalised per ng of DNA or copy number of the 16S rRNA gene. One-way analysis of variance (ANOVA) and Tukey's post-hoc tests (SPSS Statistics for Windows v.19.0; IBM Corp., Armonk, NY) were used to assess statistically significant differences $(P<0.05)$. Possible correlations between antimicrobial residues, indicator genes and bacterial populations were assessed based on Redundancy Analysis (RDA). Correlations between the variation in the copy number of each indicator gene normalised by ng of DNA or by copy number of the 16S rRNA gene (these ratios being considered the environmental variables) and the variations in the abundance of antibioticresistant cultivable bacteria and the DGGE profiles were assessed.

In addition, the concentration of antimicrobial residues was used as an environmental variable to test the variance of the normalised copy number of each indicator gene. The significance of the components was evaluated with a Monte Carlo permutation test, and only variables significantly $(P<0.05)$ explaining the observed variation were considered. Bacterial removal rates $(r)$ after wastewater treatment were estimated as:

$$
r=100-100 \times \frac{a}{b}
$$

where $a$ and $b$ represent $\mathrm{CFU} / \mathrm{mL}$ or the $16 \mathrm{~S}$ rRNA gene copy number/mL in TWW and RWW, respectively.

\section{Results}

\subsection{Variations in the abundance of indicator genes}

As estimated based on the 16S rRNA gene copy number per ng of total DNA, bacterial DNA was more abundant in HE and RWW than in the final effluent of the UWTP $(P<0.05)$ (Fig. 1). In the final effluent (TWW), the copy number of $16 \mathrm{~S}$ rRNA gene per ng of total DNA was approximately one logarithmic cycle lower than in RWW, an indication of the removal of bacteria during wastewater treatment. Considering the four indicator genes analysed, intI1 was the most abundant in all types of water, with identical prevalence whether its abundance was estimated per ng of DNA or per copy number of the $16 \mathrm{~S}$ rRNA gene $(P>$ 0.05) (Fig. 1). In contrast, the prevalence of the three indicator genes marA, blaTEM and vanA was significantly higher in HE than in RWW, either considering per ng of DNA or per copy number of the 16S rRNA gene. Comparison of the 
prevalence of these three genes in RWW and TWW showed that wastewater treatment may not remove all resistance determinants with the same efficiency. Comparing RWW and TWW, it was observed that the relative abundance of blaTEM (ratio blaTEM/16S rRNA) decreased $(P<0.05$ ), that of vanA (ratio vanA/ 16S rRNA) did not vary $(P>0.05)$ and that of marA (marA/16S rRNA) increased

$(P<0.05)$ (Fig. 1). In the same way, different indicator genes prevailed among bacteria (per copy number of $16 \mathrm{~S}$ rRNA gene) in the types of water analysed. The abundance of these genes (normalised by copy number of the 16S rRNA gene) could be ranked as intI $>$ blaTEM $>\operatorname{marA}>$ vanA in HE and RWW and intI1 > marA $>$ blaTEM $>$ vanA in TWW (Fig. 1).

\subsection{Possible correlations between indicator genes and other parameters}

To assess factors that may be correlated with the observed variations of the indicator genes, RDA was conducted. Variation in the abundance of cultivable bacteria (on PCA, GSP and $\mathrm{mFC}$, or on these media supplemented with amoxicillin or ciprofloxacin) (Supplementary Table S1) and in bacterial community composition and structure (bands of the DGGE patterns) (Supplementary Fig. S1) was analysed as a function of the abundance of each indicator gene (expressed as copy number per ng of DNA). In addition, possible relationships between variations in the abundance of these genes and the concentration of antimicrobial residues were assessed using the concentration values as environmental variables (Supplementary Table S2).

For cultivable bacteria, significant positive correlations $(P<0.05)$ were found between all bacterial groups (total heterotrophs on PCA, aeromonads on GSP and enterobacteria on $\mathrm{mFC}$ ) and the relative abundance of the different genes (Table 2). The strongest correlations were found for the genes blaTEM and vanA, with interset correlation values of 0.86 and 0.89 , respectively, with axis 1 over which the abundance of bacteria cultivable on PCA and $\mathrm{mFC}$ presented the highest cumulative fit (0.80 and 0.89, respectively) (Fig. 2A; Supplementary Fig. $\mathrm{S} 2 \mathrm{~A})$. The variation in the abundance of bacteria able to grow on PCA or GSP with ciprofloxacin or amoxicillin was also distributed over axis 1 with the strongest correlation with the variation in the abundance of blaTEM and vanA genes.

Supplementary Fig. S2 related to this article can be found, in the online version, at doi:10.1016/j.jgar.2014.10.001.

For DGGE profiles, RDA showed the distribution of bands over axis 1, with which the variations in the abundance of the tested genes were poorly correlated (Fig. 2B; Supplementary Fig. S2B). However, significant positive correlations were found between the genes vanA and blaTEM and the bacterial populations represented by bands 12, 24 and 25 (interset correlation with axis 2, 0.81 and 0.84 , respectively). These bands, which presented the highest cumulative fit over axis 2 (B12, 0.56; B24, 0.43; and B25, 0.40), suggested the presence of 
bacteria closely related to Bacteroides (closest neighbour, accession no. JQ083405.1; 100\% similarity), Aeromonas (closest neighbour, accession no. KC906261.1; 100\% similarity) and Clostridium (closest neighbour, accession no. AB79342 1.1; 99\% similarity), respectively.

To assess whether antimicrobial residues and the indicator genes could have a common source, the first were used as environmental variables and the second as biological species, searching for possible strong correlations. It was observed that vanA, blaTEM and intI1 presented significant positive correlations (cumulative fit with axis 1 of $0.66,0.63$ and 0.31 , respectively) with the concentration of tetracycline and sulfamethoxazole (interset correlation with axis 1 of 0.61 and 0.60, respectively) (Fig. 2C; Supplementary Fig. S2C). For the other chemical contaminants examined, no significant correlations were observed. In general, the same patterns of correlation were observed when the analysis was made per 16S rRNA gene copy number instead of per ng of DNA (Supplementary Fig. S3; Table 2).

Supplementary Fig. S3 related to this article can be found, in the online version, at doi:10.1016/j.jgar.2014.10.001.

Finally, analysis of the correlation between the different indicator genes would suggest a possible common source of contamination or parallel paths of dissemination. The strongest correlation was found between the abundance of blaTEM and vanA (Pearson's $r^{2}=0.91$ ), followed by blaTEM and intI1 (Pearson's $r^{2}=0.71$ ) (Table 2). A lower correlation value was observed between intI1 and vanA (Pearson's $r^{2}=0.63$ ) (Table 2 ).

\section{Discussion}

This study aimed to address questions that may contribute to both improve and simplify the monitoring of antibiotic resistance in wastewater and advance our understanding of antibiotic resistance ecology. One of the questions behind the experimental design was whether the selected genes presented different abundance in distinct types of wastewater or distinct fates during wastewater treatment. The ratio between the copy number of the 16S rRNA gene and the volume of wastewater allowed a comparison of the abundance of prokaryotic DNA in the different types of water, which was observed to be higher in HE and RWW than in TWW. Based on these data it was possible to estimate a removal rate of prokaryotic DNA during wastewater treatment of $93.8 \%$. This value was of the same order of magnitude as that estimated for the removal of culturable heterotrophs (96.8\%) [9]. The intI1 gene prevailed in all types of water after the 16S rRNA gene, and its abundance (normalised by ng of DNA or by 16S rRNA copy number) did not differ in HE, RWW or TWW. This fact suggests that intI1 may be stable in wastewater and not particularly prone to respond to external influences. The product of this gene is essential for gene recombination leading to insertions in the variable region of class 1 integrons. However, intI1 is part of the conserved region of the integron and will be equally detected in full or in 
empty integrons, explaining that no significant variations were observed for this indicator gene. Nevertheless, this is not a consensual finding since other authors reported no alteration or an increase or decrease of the gene ratio intI1/16S rRNA after wastewater treatment [17-20]. The indicator gene marA presented a significant increase in TWW compared with RWW (marA/ng DNA or marA/16S rRNA). This gene encodes a transcriptional regulator belonging to the chromosomal mar regulon of E. coli and other Enterobacteriaceae. This regulon is involved in several resistance phenotypes associated with antibiotics (tetracycline, chloramphenicol, ampicillin, nalidixic acid and ciprofloxacin), household disinfectants, organic solvents, or oxidative stress and survival under stress conditions [21]. These attributes may confer some advantage to survive wastewater treatment, justifying the higher abundance in TWW than in RWW and also its abundance in HE.

Another question of this study was whether HE represented a relevant source of resistance genes. Indeed, $\mathrm{HE}$ was observed to be a significant source of the genes blaTEM and vanA. In particular for vanA, the abundance of this gene was more than two orders of magnitude higher in HE than in RWW (four in copy number/ng DNA and two in copy number/16S rRNA). HE discharge in RWW led to dilution of the bacteria holding these genes, with significant decreases in the ratio copy number/16S rRNA. However, for vanA, wastewater treatment did not succeed in removal of the gene relative abundance to levels significantly lower than those observed in RWW. Because vancomycin use preferentially occurs in healthcare facilities, discharge of these effluents into municipal collectors is a major public health threat. The observed persistence of the vanA gene in TWW confirms previous studies [7]. blaTEM is one of the most widespread antibiotic resistance genes in the environment [6]. In contrast to vanA, wastewater treatment led to a significant reduction of blaTEM/ng DNA or blaTEM/16S rRNA. However, TWW contained ca. 165 copies of the gene per ng of DNA, which can be considered a high dose of a resistance gene to be spread in the environment. Lachmayr et al. [6], who observed that the wastewater treatment process reduced the number of bacteria but selected for the blaTEM gene, also concluded that numbers as high as ca. 240 copies of this gene per ng of DNA could be discharged by a wastewater treatment plant. The different behaviour of both genes vanA and blaTEM during wastewater treatment may be due either to the differential survival of the host organisms and/or different stability of the genetic element, although a methodological bias cannot be excluded.

The search for correlations between the selected indicator genes and the variations in the cultivable bacteria, bacterial community and antimicrobial residues was made to infer possible selective pressures and to contribute to elucidating the ecology of antibiotic resistance. The abundance of bacteria, expressed as the copy number of $16 \mathrm{~S}$ rRNA/ng DNA, was significantly correlated with most of the variables considered in this study. However, the strongest correlations were observed with the indicator genes blaTEM and vanA. The abundance of these two genes was also highly correlated $\left(r^{2}=0.91\right)$ and was 
observed to be associated with the concentration of tetracycline and sulfamethoxazole, suggesting that all are discharged by a common source. The genes bla $a_{\text {TEM }}$ and vanA also presented strong correlations with cultivable bacteria, mainly total heterotrophs $(\mathrm{PCA})$ and coliforms $(\mathrm{mFC})$. This correlation can be interpreted as the result of bacteria harbouring these genes being discharged simultaneously with cultivable bacteria that can grow on PCA or mFC. For instance, vanA occurs mainly in enterococci, which co-inhabit with coliforms and, expectedly, are excreted simultaneously into sewage. Indeed, a correlation value of 0.92 was observed between the ratio vanA/ng DNA and CFU on $\mathrm{mFC}$, on which mainly coliforms grow (Table 2). Curiously, blaTEM was not correlated with the abundance of amoxicillin-resistant coliforms, suggesting that this is probably not the most common resistance gene in that bacterial population. This fact may suggest that other bla genes such as blaCTX-M can be more adequate indicators than blaTEM. Another interesting observation was the correlation between the gene copy number marA/ng DNA and CFU on mFC supplemented with ciprofloxacin. Curiously, in a previous study it was demonstrated that ciprofloxacin-resistant E. coli (mostly recovered on $\mathrm{mFC}$ ) were significantly more prevalent in TWW than in RWW [3]. Although no evidence was gathered for a possible relationship between the mar locus and ciprofloxacin resistance in $E$. coli, an increased fitness due to the combination of chromosomal mutations in the genes gyrA, parC and marR of that locus was reported previously [22].

The strongest correlations with the bacterial community members were observed for the resistance genes blaTEM and vanA and the populations represented by bands 12, 24 and 25 that comprised bacteria of the groups Aeromonas, Clostridium and Bacteroides (Fig. 2; Table 2). Similar to the genes blaTEM and $v a n A$, these populations were also, in general, more abundant in HE or in RWW than in TWW (Supplementary Fig. S1). Members of the genus Aeromonas and the phyla Bacteroidetes and Firmicutes may harbour blaTEM, although they are not the most common host of this gene, a role attributed to Enterobacteriaceae. The vanA gene is normally only reported in enterococci but may also occur in other Firmicutes, including staphylococci [23].

In summary, the genes surveyed presented different patterns of variation in $\mathrm{HE}$ and RWW and TWW of the UWTP as well as distinct patterns of correlation with other variables, suggesting their potential as indicator genes to monitor and assess the ecology of resistance in wastewater habitats. However, it is arguable that other genes such as blaCTX-M, mecA or qac genes also widely distributed in wastewater may be also interesting indicators.

\section{Funding}

This work was supported by national funds from Fundação para a Ciência e a Tecnologia (FCT) through projects PEst-OE/EQB/ LA0016/2011 and PTDC/AACAMB/113840/2009 and grant SFRH/ BD/70986/2010. 


\section{Competing interests}

None declared.

Ethical approval

Not required.

\section{Acknowledgments}

The authors acknowledge the EU for support by Cost Action 'TD0803: Detecting evolutionary hot spots of antibiotic resistances in Europe (DARE)'; the engineers at the hospital facilities and wastewater treatment plant for their support; and Dr P. Teixeira for the Enterococcus faecalis ATCC 17050 for detection of the vanA gene.

\section{References}

[1] Vaz-Moreira I, Nunes OC, Manaia CM. Bacterial diversity and antibiotic resistance in water habitats: searching the links with the human microbiome. FEMS Microbiol Rev 2014;38:761-78.

[2] Martinez JL. Environmental pollution by antibiotics and by antibiotic resistance determinants. Environ Pollut 2009;157:2893-902.

[3] Novo A, Andre S, Viana P, Nunes OC, Manaia CM. Antibiotic resistance, antimicrobial residues and bacterial community composition in urban waste- water. Water Res 2013;47:1875-87.

[4] Martinez JL. The role of natural environments in the evolution of resistance traits in pathogenic bacteria. Proc Biol Sci 2009;276:2521-30.

[5] Goldstein C, Lee MD, Sanchez S, Hudson C, Phillips B, Register B, et al. Incidence of class 1 and 2 integrases in clinical and commensal bacteria from livestock, companion animals, and exotics. Antimicrob Agents Chemother 2001;45: 723-6.

[6] Lachmayr KL, Kerkhof LJ, Dirienzo AG, Cavanaugh CM, Ford TE. Quantifying nonspecific TEM b-lactamase (blaTEM) genes in a wastewater stream. Appl Environ Microbiol 2009;75:203-11.

[7] Varela AR, Ferro G, Vredenburg J, Yanik M, Vieira L, Rizzo L, et al. Vancomycin resistant enterococci: from the hospital effluent to the urban wastewater treatment plant. Sci Total Environ 2013;451:155-61.

[8] Castiglioni S, Pomati F, Miller K, Burns BP, Zuccato E, Calamari D, et al. Novel homologs of the multiple resistance regulator marA in antibioticcontaminated environments. Water Res 2008;42:4271-80.

[9] Varela AR, André S, Nunes OC, Manaia CM. Insights into the 
relationship between antimicrobial residues and bacterial populations in a hospital-urban wastewater treatment plant system. Water Res 2014;54:327-36.

[10] Denman SE, McSweeney CS. Development of a real-time PCR assay for moni- toring anaerobic fungal and cellulolytic bacterial populations within the rumen. FEMS Microbiol Ecol 2006;58:572-82.

[11] Bibbal D, Dupouy V, Ferre JP, Toutain PL, Fayet O, Prere MF, et al. Impact of three ampicillin dosage regimens on selection of ampicillin resistance in Enterobacteriaceae and excretion of blaTEM genes in swine feces. Appl Environ Microbiol 2007;73:4785-90.

[12] Volkmann H, Schwartz T, Bischoff P, Kirchen S, Obst U. Detection of clinically relevant antibiotic-resistance genes in municipal wastewater using real-time PCR (TaqMan). J Microbiol Methods 2004;56:277-86.

[13] DiPersio JR, Deshpande LM, Biedenbach DJ, Toleman MA, Walsh TR, Jones RN. Evolution and dissemination of extended-spectrum b-lactamaseproducing Klebsiella pneumoniae: epidemiology and molecular report from the SENTRY Antimicrobial Surveillance Program (1997-2003). Diagn Microbiol Infect Dis

2005;51:1-7.

[14] Dutka-Malen S, Evers S, Courvalin P. Detection of glycopeptide resistance genotypes and identification to the species level of clinically relevant enterococci by PCR. J Clin Microbiol 1995;33:1434.

[15] Lopes AR, Faria C, Prieto-Fernandez A, Trasar-Cepeda C, Manaia CM, Nunes OC. Comparative study of the microbial diversity of bulk paddy soil of two rice fields subjected to organic and conventional farming. Soil Biol Biochem 2011;43:115-25.

[16] Brankatschk R, Bodenhausen N, Zeyer J, Burgmann H. Simple absolute quan- tification method correcting for quantitative PCR efficiency variations for microbial community samples. Appl Environ Microbiol 2012;78:4481-9.

[17] Chen $H$, Zhang $M$. Occurrence and removal of antibiotic resistance genes in municipal wastewater and rural domestic sewage treatment systems in eastern China. Environ Int 2013;55:9-14.

[18] Ghosh S, Ramsden SJ, LaPara TM. The role of anaerobic digestion in control- ling the release of tetracycline resistance genes and class 1 integrons from municipal wastewater treatment plants. Appl Microbiol Biotechnol 2009;84: 791-6.

[19] LaPara TM, Burch TR, McNamara PJ, Tan DT, Yan M, Eichmiller JJ. Tertiary- treated municipal wastewater is a significant point source of antibiotic resistance genes into Duluth-Superior Harbor. Environ Sci Technol 2011;45: 9543-9.

[20] Stalder T, Barraud O, Jove T, Casellas M, Gaschet M, Dagot C, et al. Quantitative and qualitative impact of hospital effluent on dissemination of the integron pool. ISME J 2014;4:768-77.

[21] Alekshun MN, Levy SB. The mar regulon: multiple resistance to antibiotics and other toxic chemicals. Trends Microbiol 1999;7:410-3. 
[22] Andersson DI, Hughes D. Antibiotic resistance and its cost: is it possible to reverse resistance? Nat Rev Microbiol 2010;8:260-71.

[23] Guardabassi L, Perichon B, van Heijenoort J, Blanot D, Courvalin P. Glycopep- tide resistance vanA operons in Paenibacillus strains isolated from soil. Antimicrob Agents Chemother 2005;49:4227-33.
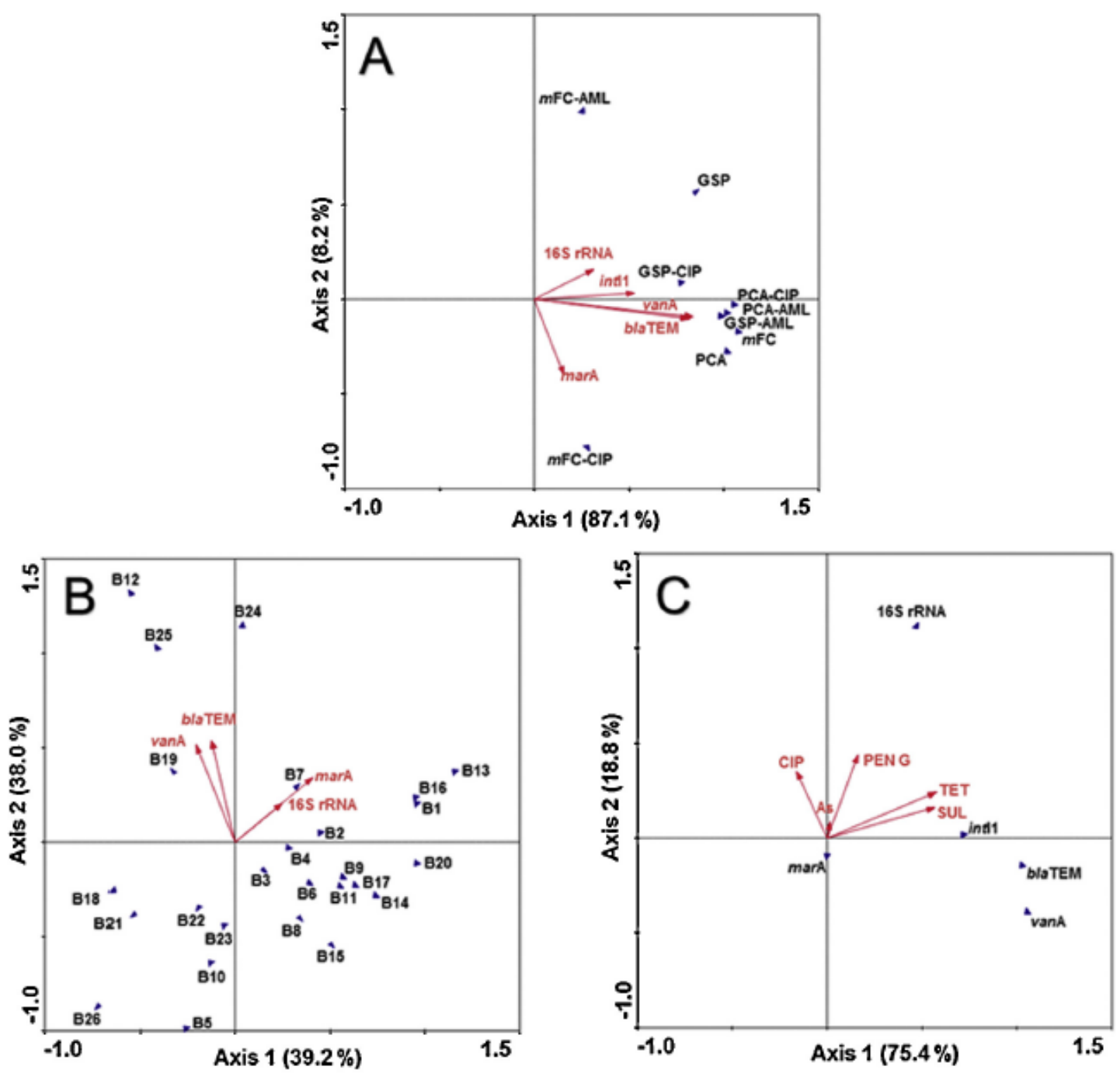

Fig. 1. Abundance of genes of interest in hospital effluent (HE) and in the raw wastewater (RWW) and treated wastewater (TWW) of the municipal wastewater treatment plant, normalised by ng of DNA and by $16 \mathrm{~S}$ rRNA gene copies. The quantities of total DNA per $\mathrm{mL}$ of sample were: for $\mathrm{HE}$ in October $908.4 \mathrm{ng}$, in January $427.1 \mathrm{ng}$, in February

$358.9 \mathrm{ng}$ and in June $566.7 \mathrm{ng}$; for RWW in October $650.9 \mathrm{ng}$, in January $609.7 \mathrm{ng}$ and in February $637.6 \mathrm{ng}$; and for TWW in October $87.7 \mathrm{ng}$, in January $127.6 \mathrm{ng}$ and in February $159.6 \mathrm{ng}$. a,b,c Indicate significantly $(P$ $<0.05)$ different groups for the same ratio comparing the different types of water. 


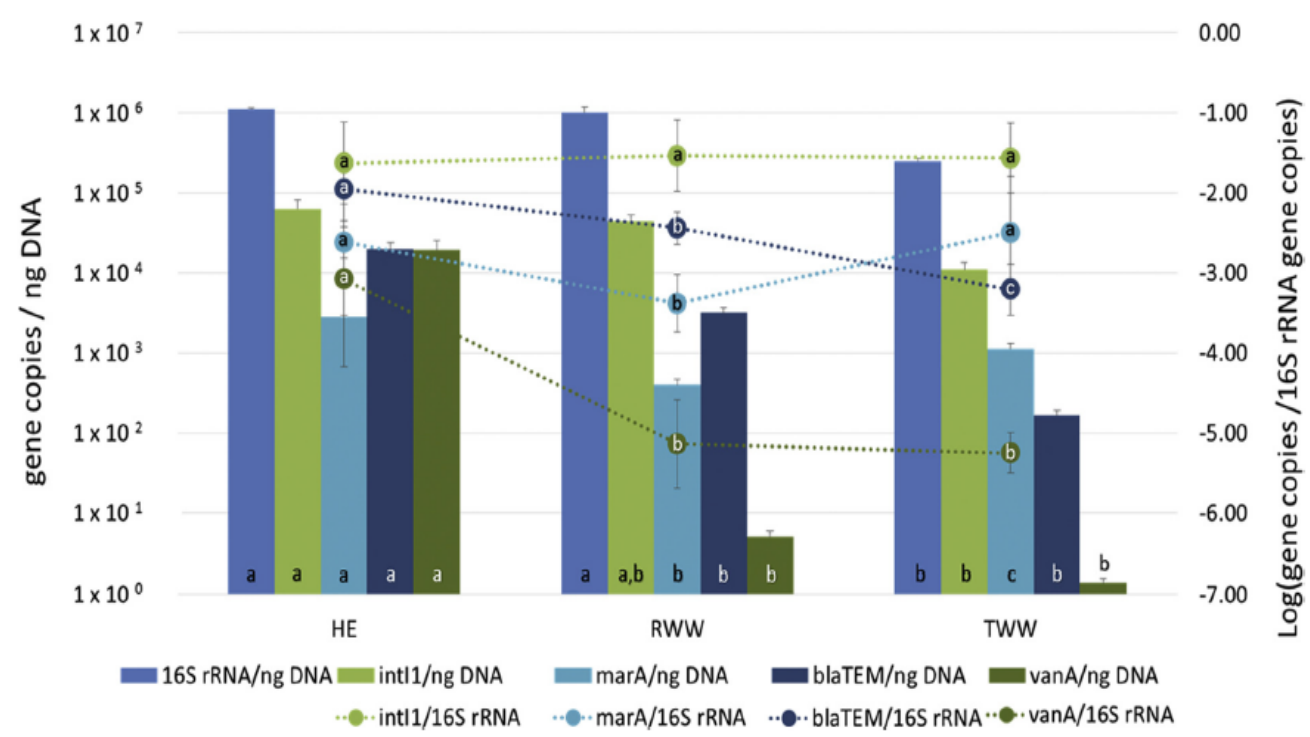

Fig. 2. Relationships between environmental variables (genes of interest and antibiotic concentrations, per ng of DNA), total and resistant heterotrophic bacterial counts, and denaturing gradient gel electrophoresis (DGGE) profile bands. (A) Redundancy Analysis (RDA) distance biplot of resistant heterotrophic bacteria constrained by genes of interest; (B) RDA distance biplot of DGGE profile bands constrained by genes of interest; and (C) RDA distance biplot of genes of interest constrained by antibiotic concentration. Explanatory variables are denoted as bold red arrows. Variables with non-significant correlations $(P$ $>0.05$ ) were not included in these analyses (See also

Supplementary Fig. S1). PCA, plate count agar; GSP, glutamate-starchphenol red agar; $\mathrm{mFC}, \mathrm{m}$-faecal coliform agar; AML, amoxicillin; CIP, ciprofloxacin; PEN G, penicillin G;

TET, tetracycline; SUL, sulfamethoxazole. (For interpretation of the references to colour in this figure legend, the reader is referred to the web version of this article.) 
Table 1

Real-time PCR primer sequences and reaction conditions.

\begin{tabular}{|c|c|c|c|c|c|}
\hline Target gene & Primer & Sequence & Conditions & Efficiency (\%) & Reference \\
\hline \multirow[t]{2}{*}{ 16S rRNA } & $1114 \mathrm{~F}$ & CGGCAACGAGCGCAACCC & \multirow{2}{*}{$\begin{array}{l}95^{\circ} \mathrm{C} \text { for } 10 \mathrm{~min}(1 \mathrm{cycle}) ; 95^{\circ} \mathrm{C} \text { for } 15 \mathrm{~s}, 55^{\circ} \mathrm{C} \text { for } 20 \mathrm{~s} \\
\text { and } 72{ }^{\circ} \mathrm{C} \text { for } 10 \mathrm{~s} \text { ( } 35 \text { cycles) }\end{array}$} & \multirow[t]{2}{*}{100} & \multirow[t]{2}{*}[10]{} \\
\hline & $1275 \mathrm{R}$ & CCATTGTAGCACGTGTGTAGCC & & & \\
\hline \multirow[t]{2}{*}{$b l a_{\mathrm{TEM}}$} & blaTEM-F & TTCCTGTTTTTGCTCACCCAG & \multirow{2}{*}{$\begin{array}{l}95^{\circ} \mathrm{C} \text { for } 10 \mathrm{~min}(1 \mathrm{cycle}) ; 95^{\circ} \mathrm{C} \text { for } 15 \mathrm{~s}, 60^{\circ} \mathrm{C} \text { for } 30 \mathrm{~s} \\
\left.\text { and } 72{ }^{\circ} \mathrm{C} \text { for } 10 \mathrm{~s} \text { ( } 40 \text { cycles }\right)\end{array}$} & \multirow[t]{2}{*}{96} & \multirow[t]{2}{*}{ [11] } \\
\hline & blaTEM-R & CTCAAGGATCTTACCGCTGTTG & & & \\
\hline \multirow[t]{2}{*}{ marA } & EmarAF & ACGGAAATCGCGCAAAAG & \multirow{2}{*}{$\begin{array}{l}95^{\circ} \mathrm{C} \text { for } 5 \mathrm{~min}(1 \mathrm{cycle}) ; 95^{\circ} \mathrm{C} \text { for } 3 \mathrm{~s} \text { and } 60^{\circ} \mathrm{C} \text { for } 30 \mathrm{~s} \\
\text { (40 cycles) }\end{array}$} & \multirow[t]{2}{*}{95} & \multirow[t]{2}{*}{ [8] } \\
\hline & EmarAR & CCAGATAGAGTATCGGCTCGTTACTT & & & \\
\hline \multirow[t]{2}{*}{ intl1 } & intI1-F & CCTCCCGCACGATGATC & \multirow{2}{*}{$\begin{array}{l}95^{\circ} \mathrm{C} \text { for } 10 \mathrm{~min}(1 \mathrm{cycle}) ; 95^{\circ} \mathrm{C} \text { for } 15 \mathrm{~s}, 55^{\circ} \mathrm{C} \text { for } 30 \mathrm{~s} \\
\text { and } 72^{\circ} \mathrm{C} \text { for } 10 \mathrm{~s} \text { ( } 40 \text { cycles) }\end{array}$} & \multirow[t]{2}{*}{94} & \multirow[t]{2}{*}{ [5] } \\
\hline & intl1-R & TCCACGCATCGTCAGGC & & & \\
\hline \multirow[t]{2}{*}{ vanA } & vanA3FP & CTGTGAGGTCGGTTGTGCG & \multirow{2}{*}{$\begin{array}{l}95^{\circ} \mathrm{C} \text { for } 5 \mathrm{~min}(1 \mathrm{cycle}) ; 95^{\circ} \mathrm{C} \text { for } 3 \mathrm{~s} \text { and } 60^{\circ} \mathrm{C} \text { for } 30 \mathrm{~s} \\
\text { (40 cycles) }\end{array}$} & \multirow[t]{2}{*}{98} & \multirow[t]{2}{*}[12]{} \\
\hline & vanA3RP & TTTGGTCCACCTCGCCA & & & \\
\hline
\end{tabular}




\section{Table 2}

Parameters exhibiting Pearson's correlation values $\left(r^{2}\right)$ of $>0.5$ for at least one of the genes analysed. Only those strongly correlated with environmental variables in the Redundancy Analysis were considered for the discussion.

\begin{tabular}{|c|c|c|c|c|c|c|c|c|c|c|c|c|c|c|c|c|c|c|c|c|c|}
\hline \multirow[t]{2}{*}{ Gene/ng DNA } & \multicolumn{4}{|c|}{ Indicator } & \multicolumn{8}{|c|}{ Total and resistant heterotrophic bacteria } & \multicolumn{6}{|c|}{ DGGE profile bands } & \multicolumn{3}{|c|}{ Antimicrobial residues } \\
\hline & $b l a_{\mathrm{TEM}}$ & intl1 & $\operatorname{mar} A$ & vanA & PCA & GSP & $\mathrm{mFC}$ & PCA AML & PCA CIP & GSP AML & GSP CIP & $\mathrm{mFC} \mathrm{CIP}$ & B2 & B12 & B13 & B16 & B24 & B25 & TET & PEN & SUL \\
\hline $16 \mathrm{~S}$ rRNA & $0.34^{* *}$ & $0.38^{* *}$ & $0.23^{\circ}$ & 0.19 & $0.21^{\circ}$ & $0.45^{*}$ & $0.28^{* \prime}$ & $0.27^{\circ}$ & $0.39^{* *}$ & $0.27^{*}$ & 0.14 & $0.31^{*}$ & $0.51^{*}$ & 0.12 & 0.17 & 0.06 & $0.39^{*}$ & $0.32^{* \prime}$ & $0.55^{*}$ & $0.57^{* *}$ & $0.44^{* *}$ \\
\hline$b a_{\mathrm{TEM}}$ & 1.00 & $0.71^{\circ}$ & $0.37^{\circ}$ & $0.91^{*}$ & $0.86^{* \prime}$ & $0.64^{*}$ & $0.89^{* \prime}$ & $0.83^{* *}$ & $0.87^{* *}$ & $0.82^{*}$ & $0.64^{*}$ & $0.34^{* \prime}$ & 0.04 & $0.77^{*}$ & 0.03 & -0.05 & $0.63^{*}$ & $0.61^{*}$ & $0.56^{*}$ & 0.09 & $0.58^{*}$ \\
\hline intl1 & & 1.00 & 0.20 & $0.63^{*}$ & $0.52^{* *}$ & $0.47^{\circ}$ & $0.56^{*}$ & $0.53^{* *}$ & $0.55^{*}$ & $0.54^{*}$ & $0.45^{*}$ & 0.16 & 0.02 & $0.38^{*}$ & -0.11 & -0.12 & $0.40^{\circ}$ & $0.41^{*}$ & $0.41^{*}$ & 0.12 & $0.44^{\prime \prime}$ \\
\hline marA & & & 1.00 & $0.33^{*}$ & $0.23^{\circ}$ & -0.04 & $0.21^{\circ}$ & 0.17 & 0.21 & 0.18 & 0.01 & $0.55^{\circ}$ & 0.04 & $0.25^{\circ}$ & $0.69^{*}$ & $0.59^{*}$ & $0.36^{*}$ & 0.11 & -0.03 & -0.18 & -0.01 \\
\hline vanA & & & & 1.00 & $0.89^{*}$ & $0.65^{\circ}$ & $0.92^{*}$ & $0.87^{\circ}$ & $0.88^{*}$ & 0.84 " & 0.65 & $0.25^{\circ}$ & -0.07 & $0.82^{*}$ & -0.05 & -0.05 & $0.60^{\circ}$ & $0.65^{\circ}$ & $0.54{ }^{*}$ & 0.03 & $0.51^{*}$ \\
\hline
\end{tabular}

PCA, plate count agar; GSP, glutamate-starch-phenol red agar; mFC, m-faecal coliform agar; AML, amoxicillin; CIP, ciprofloxacin; DGGE, denaturing gradient gel electrophoresis; TET, tetracycline; PEN, penicillin G; SUL, sulfamethoxazole.

Shadowed cells represent accordance in significance $(P<0.05)$ of significant correlations observed when calculation were made with gene copies/ng DNA or gene copies/16S rRNA gene copies

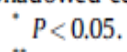

" $P<0.01$. 